\title{
Fully automatic measurements of axial vertebral rotation for assessment of spinal deformity in idiopathic scoliosis
}

\author{
Daniel Forsberg ${ }^{1,2,5}$, Claes Lundström ${ }^{2,5}$, Mats

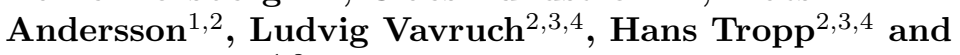 \\ Hans Knutsson ${ }^{1,2}$ \\ ${ }^{1}$ Department of Biomedical Engineering, Linköping University, Sweden \\ 2 Center for Medical Image Science and Visualization, Linköping University, \\ Sweden \\ ${ }^{3}$ Department of Clinical and Experimental Medicine, Linköping University, \\ Sweden \\ ${ }^{4}$ Spine Center, University Hospital, Linköping, Sweden \\ ${ }^{5}$ Sectra, Linköping, Sweden \\ E-mail: dafor@imt.liu.se
}

\begin{abstract}
Reliable measurements of spinal deformities in idiopathic scoliosis are vital, since they are used for assessing the degree of scoliosis, deciding upon treatment and monitoring the progression of the disease. However, commonly used two dimensional methods (e.g. the Cobb angle) do not fully capture the three dimensional deformity at hand in scoliosis, of which axial vertebral rotation (AVR) is considered to be of great importance. There are manual methods for measuring the AVR, but they are often time-consuming and related with a high intra- and inter-observer variability. In this paper, we present a fully automatic method for estimating the AVR in images from computed tomography. The proposed method is evaluated on four scoliotic patients with 17 vertebrae each and compared with manual measurements performed by three observers using the standard method by Aaro-Dahlborn. The comparison shows that the difference in measured AVR between automatic and manual measurements are on the same level as the interobserver difference. This is further supported by a high intraclass correlation coefficient (0.971-0.979), obtained when comparing the automatic measurements with the manual measurements of each observer. Hence, the provided results and the computational performance, only requiring approximately ten to fifteen seconds for processing an entire volume, demonstrate the potential clinical value of the proposed method.
\end{abstract}

\section{Introduction}

For a long time assessments of spinal deformities have relied on two dimensional (2D) radiographs and manual methods, and still do to some extent, although three dimensional (3D) data from computed tomography (CT), magnetic resonance imaging (MRI) and more automated methods are available. In this paper, we propose a fully automatic method, for the assessment of spinal deformity in idiopathic scoliosis, measuring the axial vertebral rotation in CT data.

Scoliosis is traditionally defined as an abnormal lateral curvature of the spine, observed in the coronal plane. In most cases the cause of scoliosis is unknown, 


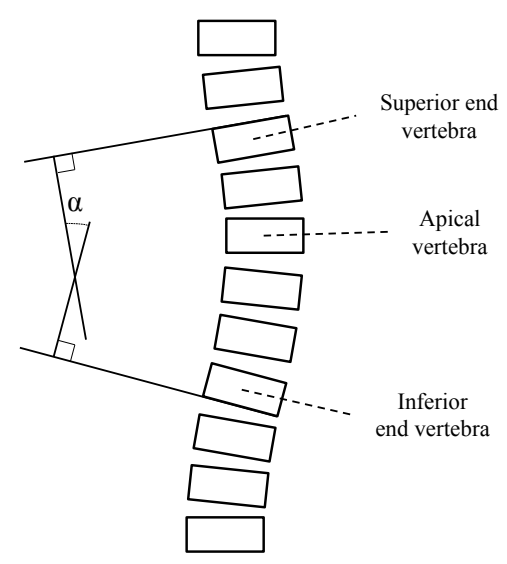

Figure 1. Schematic example of the Cobb angle, measured as the angle between the two lines drawn parallel to the upper and the lower endplates of the two end vertebrae.

i.e. idiopathic scoliosis. Scoliosis is typically categorized depending on curvature type (C- or S-like), location (thoracic, lumbar or thoracolumbar) and age of onset (infantile, juvenile, adolescent or adult). The deformity of the spinal curvature is often detected during a standard physical examination. For assessing the severity of the deformity, an anterior-posterior radiography is used where the Cobb angle is measured (Cobb 1948). The Cobb angle is defined as the angle between the two lines drawn parallel to the superior endplate of the superior end vertebra and parallel to the inferior endplate of the inferior end vertebra, see figure 1. The end vertebrae of a curve are defined as the vertebrae that are the most tilted in the curve, as observed in the frontal plane. There exist several other methods for evaluating both coronal and sagittal curvature of the spine, but the Cobb angle remains the most frequently used. A recent review of various methods is found in the work by Vrtovec, Pernuš and Likar (2009).

The choice of treatment depends on, among other factors, age of onset, gender, skeletal maturity, the Cobb angle and the estimated progression rate of the Cobb angle. For less severe cases, with remaining growth and a scoliotic curve of 25 to 40 degrees, bracing is usually offered as a treatment. When the curvature is more pronounced, surgery is necessary in order to stop progression and to correct some deformation. A fusion is performed, always including the primary curve and sometimes also the secondary compensatory curve. Hence, the Cobb angle plays an important role when selecting the appropriate treatment. The different intervals of the Cobb angle, guiding the choice of treatment, are here defined in accordance with the intervals used at the surgical department of the local hospital, which are based upon Templin and Sarwark (2008).

A scoliotic deformity is always 3D, because it also includes an axial rotation of the vertebrae and not only a displacement and rotation in the coronal plane. The apical vertebra of the primary curve is always the most rotated. This axial rotation limits the use of the Cobb angle because it only measures on the projection of the curve onto a 2D plane. Also, more recent research has shown that the axial vertebral rotation (AVR) is more relevant for both understanding the underlying causes of idiopathic 
scoliosis, but also for deciding upon treatment and monitoring the progression of the disease (Skalli et al. 1995, Kuklo et al. 2005, Heidari et al. 2006). Hence, there is a need for other measurement methods that can better assess the full 3D deformity of the spine in scoliosis, i.e. measure the axial vertebral rotation.

Over the years, a number of different methods for measuring AVR have been proposed, where most of them are manual methods using either 2D data or 3D data. Examples of methods for AVR measurements are the methods by Cobb, Stokes and Aaro-Dahlborn. These and other commonly used manual methods are described in Lam et al. (2008) and Vrtovec, Pernu and Likar (2009). Manual methods, however, often suffer from being time-consuming, complex and related with a relatively high intra- and inter-observer variability. Hence, there is an interest in developing more automatic methods.

Rogers et al. (2002) presented a method for measurement of inter-vertebral rotation in the lumbar spine, based upon image registration, applicable to both CT and MRI, and later evaluated in (Rogers et al. 2005). To find the inter-vertebral rotation, the observer would select the two axial images considered to show as much as possible of the vertebral processes, and then define the centre of rotation in both of the images. The rotation is then estimated by rotational registration, maximizing the correlation between the two images. Adam and Askin (2006) developed a semiautomatic method for measuring the vertebral rotation. Their method was based on the observer selecting a suitable axial image, defining a region of interest around the vertebra and thereafter applying a threshold for bone and estimating the centroid. The symmetry ratio was maximized to find the rotation of the vertebra around its centroid. Due to the threshold, this method is limited to CT images. Another method limited to CT images was presented by Kouwenhoven et al. (2006). Here the observer was asked to select an axial image depicting each vertebra with the pedicles clearly visible. The vertebra and the spinal canal were then segmented in each image using region growing, from which their respective centroids were estimated. The actual rotation was then estimated as the angle between the line running through the centroids of the vertebra and the spinal canal, and the reference line between the centroids of the sternum and the spinal canal at the level of vertebra T5. Vrtovec (2008) presented a modality-independent method for determining the vertebral position and rotation in 3D, based upon earlier work by Vrtovec et al. (2007). The method utilizes registration of symmetry to find the position and rotation of the vertebrae. However, the observer would still have to initialize the method by defining the size of the symmetrical masks to use and to provide an initial location of each vertebra. This method was evaluated in (Vrtovec et al. 2010) on MRI data.

The methods by Rogers et al. (2002), Adam and Askin (2006), and Kouwenhoven et al. (2006) are limited in measurement accuracy, since they only use 2D axial images when estimating the rotation, whereas the method by Vrtovec (2008) utilizes the full 3D information available. However, all four methods require more or less manual interaction, and therefore intra- and inter-observer variability is still likely to occur.

The purpose of this paper is to propose a method that overcomes some of the limitations of the previously presented computerized methods. The method is fully automatic, measures the axial vertebral rotation in 3D based on CT data and is sufficiently computationally efficient to be integrated into a clinical workflow. A fully automatic method is important both for reducing or completely eliminating the intra- and inter-observer variability and for providing a method that can be used to analyse large amounts of data with minimal user interaction. As suggested in 
previous research, the axial vertebral rotation is considered to be of great importance for assessing idiopathic scoliosis and, hence, it is a measure we target our method towards. However, our method is not limited to only measure the AVR but is able to estimate the full pose of each vertebra. The computational performance is also important, especially for a method to be feasible in a clinical workflow, where time is a particularly scarce resource and efficiency is an important feature.

\section{Method}

The proposed method is based on the following steps; extraction of the spinal canal centreline, disc detection, vertebra centrepoint estimation and vertebra rotation estimation. Figure 2 provides a schematic overview of the method. To some extent we have relied on previous work by others, and in those cases our contribution is related to integrating earlier described algorithms and adapting them in order to provide a solution that is clinically feasible, i.e. fast and robust. This applies particularly to the extraction of the spinal canal centreline and the vertebra rotation estimation. Where appropriate we will refer to these previous work or provide options for alternative methods achieving similar results. In this work, we have assumed that the provided image volume to process primarily depicts the spine of a patient, however, not necessarily covering the entire spine but, at least including the lumbar vertebrae and most of the thoracic vertebrae.

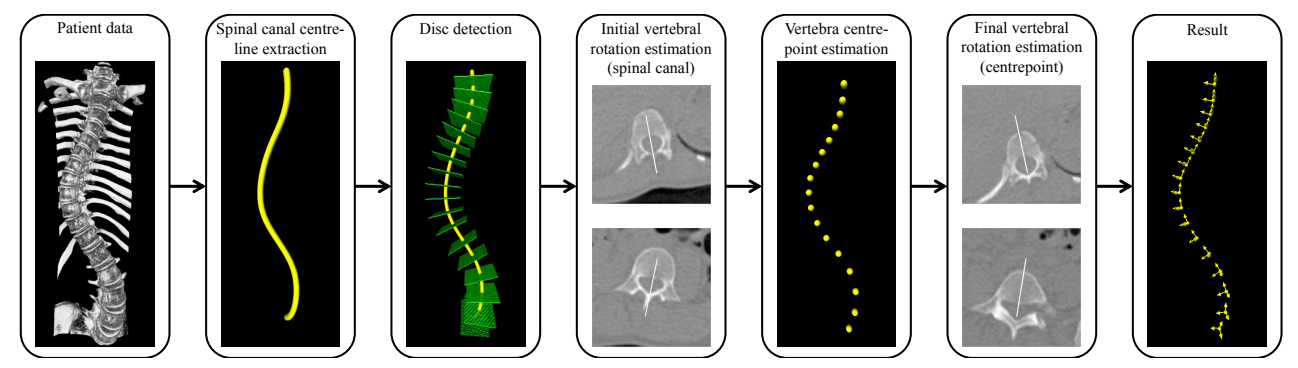

Figure 2. An overview of the proposed method for automatic measurements of AVR. Figure 5 provides more detailed images of the patient data and the end results.

\subsection{Pre-processing}

The image data was pre-processed before processing by applying a threshold at 100 Hounsefield unit (HU), to limit the influence of other tissues than bone.

\subsection{Spinal canal centreline extraction}

The first step is to extract the centreline of the spinal canal. This is achieved by utilizing the a priori knowledge that the spinal canal is essentially encircled by bone on all sides. A seed point for the spinal canal is found by extracting a subvolume of axial images in the centre of the image volume and applying a maximum intensity projection (MIP) along the caudal-cranial direction followed by a threshold for bone to create a binary map for bone. The seed point for the spinal canal is then found 
using the Hough transform for locating a circle with radius $R_{\min }<R<R_{\max }$. The found seed point is fed, as initial starting point, to the following algorithm.

(i) Generate a circle at a given starting point and with the radius $R_{\min }$ in a given axial image.

(ii) Enlarge the circle until it hits bone or its radius reaches the given size $R_{\max }$. Here bone is defined as having a HU-value larger than 300 .

(iii) If the circle hits bone, move it away from the bone, if possible, and then repeat step (ii).

(iv) Check the radius of the circle once growing has stopped.

(a) If the radius is $R_{\min }$, then we are inside bone somewhere, e.g. in the sacrum if moving in the caudal direction, and hence we should stop.

(b) If the radius is $R_{\max }$, then we are outside the spinal canal, e.g. we have reached the scull if moving in the cranial direction, and hence we should stop.

(c) Otherwise, propagate the estimated centrepoint to the next axial image and repeat from step (i).

The described procedure is executed both in the caudal and the cranial direction starting from the mid-axial image. This suggestion is based on an algorithm for spinal canal extraction described by Kim and Kim (2009). However, our approach differs by working on a single 2D image at a time, whereas Kim and Kim do the processing in $3 \mathrm{D}$ using a sphere. Here the $2 \mathrm{D}$ approach has the benefit of a significantly improved computational performance. Also our seed point detection differs from their approach. Since the assumption that the spinal canal is encircled by bone does not always hold, the robustness has to be addressed. This is achieved using a MIP of a number of surrounding axial images instead of using only a single axial image, which is employed in each iteration of the above described algorithm and not only for the seed point detection. When all images have been traversed both in the caudal and cranial direction, a curve is finally fitted to the found centrepoints using cubic B-splines with eight knots (breaks), which improves the smoothness of the extracted centreline. This for instance deals with the issue of incorrectly estimated centrepoints, which might occur when the assumption of a circular shaped spinal canal does not hold.

At this point a reasonable question is why we chose to use the Hough transform for detecting a seed point, but a different approach with the growing and moving circle for actually extracting the centreline of the spinal canal. In our work, we encountered some issues with both robustness and speed when using the Hough transform, which in the end favoured the use of the growing and moving circle. Another question related to the spinal canal extraction is the selection of HU-value in step (ii) for defining bone. The HU-value was by purpose set a bit lower than traditional HU-values defining bone. This was done to handle some issues with spongy bone in the posterior vertebral body.

Other examples of extracting the spinal canal include the works by Yao et al. (2006), using a watershed transform, by Klinder et al. (2009), where small tubeshaped segments are adapted and concatenated along the spinal canal, and by Hay et al. (2009), using stepwise morphological region growing.

\subsection{Disc detection}

The second step is to detect the approximate location of the discs between the vertebrae. This is achieved by analysing the intensity profile along the centreline 
of the spinal canal, but displaced towards the anterior of the image volume. The displacement varies depending on location, e.g. $20 \mathrm{~mm}$ at vertebra L5 and linearly decreasing to $10 \mathrm{~mm}$ at vertebra T1. Here we assume that the vertebral bodies are located in front of the spinal canal, an assumption that holds most of the time, apart from extremely severe cases of scoliosis, where the AVR can exceed 45 degrees.

The intensity profile of this line has a distinctive pattern of two high peaks surrounding a deep valley, indicating the positions of the discs. By detection of this pattern, we can estimate an approximate location of the discs and also the locations of the mid-axial images of the vertebrae, which are located in the middle of two discs. To detect the pattern, the intensity profile is filtered with a complex valued lognormal quadrature filter, a joint edge and line detection filter with centre frequency $\pi / 4$ and a relative bandwidth of 2.0 octaves, see (Granlund and Knutsson 1995) for a more detailed description of quadrature filters and their usage. The magnitude of the filtered intensity profile provides a robust indicator of where lines and edges are located in the signal. Furthermore, the magnitude of the filtered signal is searched to find its large peaks, where a peak can indicate vertebra-disc or vertebra-disc-vertebra, which sequence depends on the distance between two adjacent vertebrae. Two peaks close together, e.g. with a distance less than $15 \mathrm{~mm}$ for L5 and linearly decreasing to $5 \mathrm{~mm}$ for T1, or a single peak provides a robust indication of the location of a disc. In figure 3 , both the intensity profile with located discs and vertebrae, and the magnitude of the filtered intensity profile can be observed.

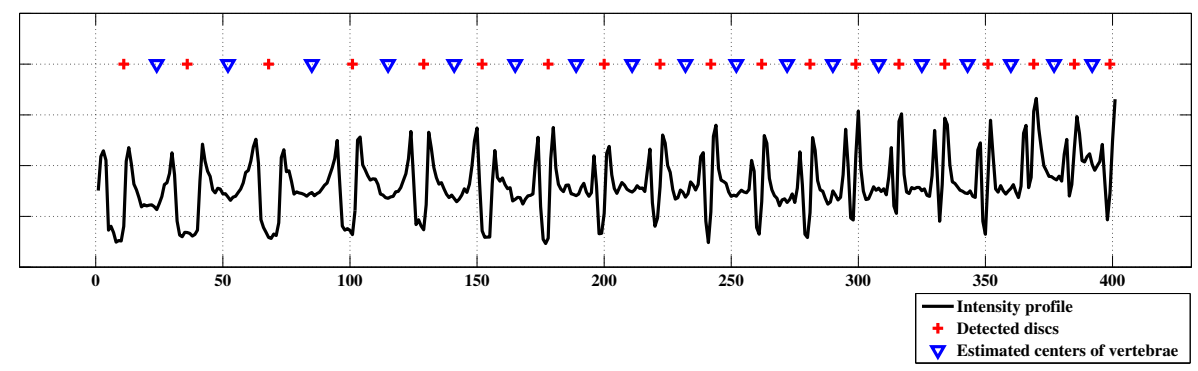

(a)

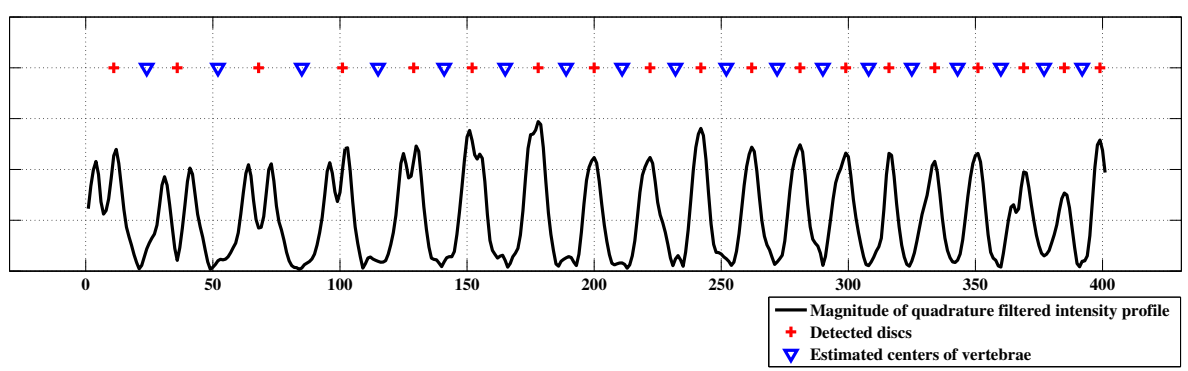

(b)

Figure 3. Example of an intensity profile (a) running through the discs and the vertebrae. Notice the distinctive pattern of the discs, i.e. a deep valley surrounded by two sharp peaks in the intensity profile. In the filtered signal (b) the disc locations are easily and robustly detected by searching for peaks with a large magnitude. 
A similar approach (analysing intensity profiles), for detecting the disc locations and the vertebra centres, is described in the work by Štern et al. (2010). They differ in their approach by analysing both the intensity profile and the gradient magnitude profile at the same time in order to detect the discs and the vertebrae centres in a robust manner. Despite our simpler approach, we still appear to achieve a result with similar robustness as in Štern et al. (2010).

\subsection{Initial vertebral rotation estimation}

Given the centreline of the spinal canal, we then estimate how each vertebra is rotated around the tangent of the spinal canal, at the approximate mid-axial images of the vertebrae, using the following algorithm.

(i) Start from the lowest detected vertebra and let $R_{0}=0^{\circ}$.

(ii) Resample an oblique plane in the centre of the vertebra, using the tangent of the spinal canal as normal vector of the plane.

(iii) Allow this plane to rotate $\pm 10^{\circ}$ around the rotation $R_{0}$ with an angular step size of $0.1^{\circ}$ and with the centrepoint of the spinal canal as rotation centre. For each rotation angle, assess the symmetry across the anterior-posterior line using the sum of absolute difference (SAD).

(iv) The rotational angle with the lowest $\mathrm{SAD}$ corresponds to the sought rotation.

(v) Proceed to the next vertebra and use the estimated rotation of the previous vertebra as $R_{0}$. Repeat from step (ii) until all vertebrae have been processed.

Vrtovec et al. (2005) has used a similar approach for rotation estimate, but where they use correlation instead of SAD for detecting symmetry. Experiments were also performed using correlation, yielding similar results, but the use of SAD was considered to be more robust, and hence became our choice. The rotation interval $\pm 10^{\circ}$, as defined in step (iii), might for the lowest vertebra have to be increased to handle large initial rotations.

\subsection{Vertebra centrepoint estimation}

To find the centrepoint of a vertebral body, we relied on the fact that the vertebral body often is symmetrical, i.e. given a point inside the vertebra and knowing the approximate orientation of the vertebra, by estimating the length, width and height of the vertebra along the axis of the given orientation, we can iteratively move the given point closer to the centre of the vertebra, as depicted in figure 4

From the previous steps, we have a rough estimate of the centrepoint of the vertebral body and its rotation. Based upon this, we resample three orthogonal planes, centred in the initial centrepoint and oriented according to the tangent of the spinal canal centreline and the estimated rotation. Three orthogonal intensity profiles, running in the anterior-posterior direction, the caudal-cranial direction and the lateral direction of the vertebra, are then extracted. These three intensity profiles are analysed to determine the point of reflective symmetry and thereby locating the centre of the vertebral body. Once all centrepoints have been found, a curve is fitted to the centrepoints, again using cubic B-splines and eight knots.

Related work for this step, i.e. localization of the centrepoint of the vertebrae, includes the already mentioned work by Stern et al. (2010) and also the very recent work by Glocker et al. (2012). Note, however, that Štern et al. (2010) report a total 


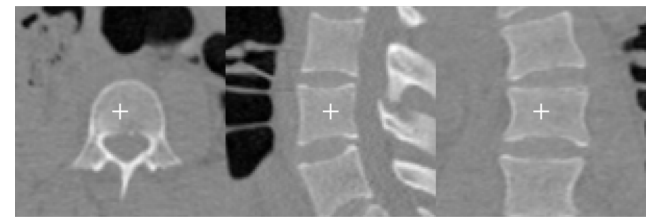

(a)

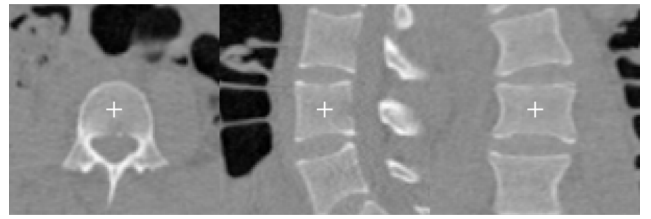

(b)

Figure 4. Three orthogonal planes (axial, sagittal and coronal) centred in the estimated centrepoint of the vertebral body, (a) depicts the initial centrepoint and (b) the finally estimated centrepoint after utilizing the reflective symmetry in the orthogonal intensity profiles running through the estimated centrepoint.

processing time ranging from minutes to hours and Glocker et al. (2012) a processing time close to two minutes.

\subsection{Final axial vertebral rotation estimation}

The final estimation of AVR is performed using the method outlined in section 2.4, but this time estimating the rotation around the centrepoint of the vertebra and using the tangent of the curve running through the centrepoints. Figure 5 depicts an example of the final result where a spine has been processed, the centrepoints of each vertebra found and the orientations of each vertebra estimated.

\section{Experiments}

To evaluate the proposed method, a study was conducted to compare it with a manual standard method. The purpose of the study was to determine whether the variability between the measurements of the automatic method and an observer is on the same level as the inter-observer variability.

\subsection{Image Data}

Four CT data sets of patients suffering from scoliosis were retrospectively gathered from the local picture archiving and communication system, all including vertebrae T1-L5. Hence, in total 68 vertebrae were analysed with corresponding AVR measurements. Included patients were in average $15 \pm 1.7$ years old at the time of the examination with an average Cobb angle of $62.9^{\circ} \pm 17.0$. Before analysis the image data was anonymized. All data sets had a resolution less than $1 \mathrm{x} 1 \mathrm{x} 1 \mathrm{~mm}^{3}$, typically anisotropic. Both the automatic and manual measurements were performed on data resampled to an isotropic resolution of $1 \times 1 \times 1 \mathrm{~mm}^{3}$ using tri-linear interpolation.

In regards to radiation dose, it should be noted that the image data was taken as a part of the routine procedure for pre-operative planning at the local hospital, using a special protocol for low-dose examinations of the spine. This protocol is especially targeted at minimizing the delivered dose while maintaining image quality, see Kalra et al. (2012). 


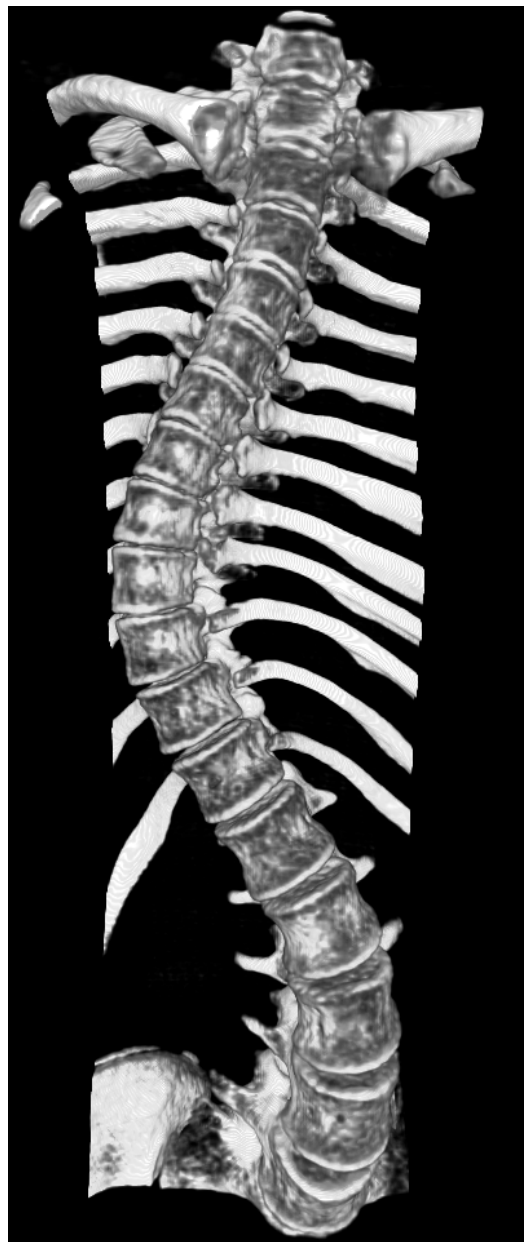

(a)

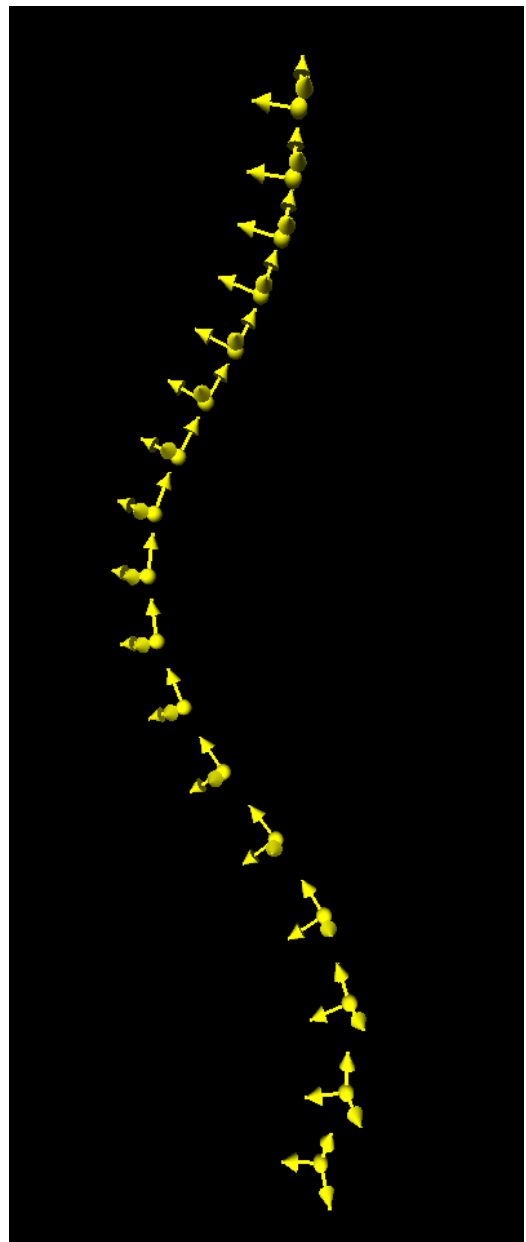

(b)

Figure 5. An example of the result after processing an image volume containing a scoliotic spine (a). In (b) the orientations of each vertebra, presented through a local coordinate system, are visualized, where the orientations are based upon the tangent of the curve running through the centrepoints of the vertebrae and the estimated axial vertebral rotations around the same.

\subsection{Observers}

Three observers were included in the study to compare the proposed automatic method with a manual method. The observers are all experienced in medical imaging (one radiologist, one orthopaedic surgeon and one graduate student in medical imaging). They performed the measurements on the patient data sets after obtaining instructions and practicing first. 


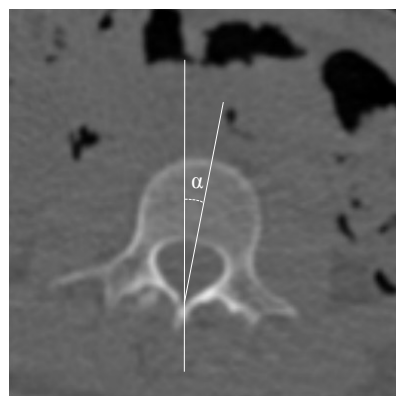

Figure 6. According to Aaro-Dahlborn, AVR is measured as the angle between a straight line through the dorsal central aspect of the vertebral foramen and the centrepoint of the vertebral body, and a line parallel to the sagittal plane.

\subsection{Manual Method}

The observers were asked to use the manual method of Aaro and Dahlborn (Aaro and Dahlborn 1981) for measuring AVR of each vertebra. This is done by measuring the angle between a straight line through the dorsal central aspect of the vertebral foramen and the centrepoint of the vertebral body, and a line parallel to the sagittal image plane of the image volume, as depicted in figure6. This method was originally developed for use in axial images, i.e. not considering the possible tilt of the vertebral body in the sagittal and/or the coronal plane. To circumvent this issue, the image data was resampled using curved planar reformation along the curve running through the centrepoints of the vertebral bodies. These centrepoints were manually marked by each observer. The measurements where then performed in oblique axial cross-sections orthogonal to the curve running through centrepoints of the vertebral bodies, where the axial cross-sections had the same axial orientation as the original axial images.

\section{Results}

The variability between the different measurements of the 68 vertebrae are graphically visualized using Bland-Altman plots (mean-difference plots), depicted in figure 7 . These plots show that the mean differences and their $95 \%$ confidence intervals (CI) between the observers are $-0.01^{\circ} \pm 4.83,0.20^{\circ} \pm 4.48$ and $0.21^{\circ} \pm 4.84$ (Observer 1 Observer 2, Observer 1 - Observer 3 and Observer 2 - Observer 3), and $-0.62^{\circ} \pm 4.83$, $-0.60^{\circ} \pm 4.43$ and $-0.81^{\circ} \pm 5.02$ between the observers and the automatic measurements (Observer 1 - Automatic, Observer 2 - Automatic and Observer 3 - Automatic). Both the mean differences and CIs are of the same magnitude when comparing manual measurements with manual and automatic measurements with manual.

To statistically compare the correlation in-between all manual measurements and automatic measurements, we employed the intraclass correlation coefficient (ICC), using a mixed two-way model with absolute agreement and single measurements. Obtained intraclass correlation coefficients and their 95\% confidence intervals are given in table 1. All comparisons gave relatively high ICC values, between 0.971 and 0.979 , suggesting a high level of agreement between the manual and the automatic measurements. 

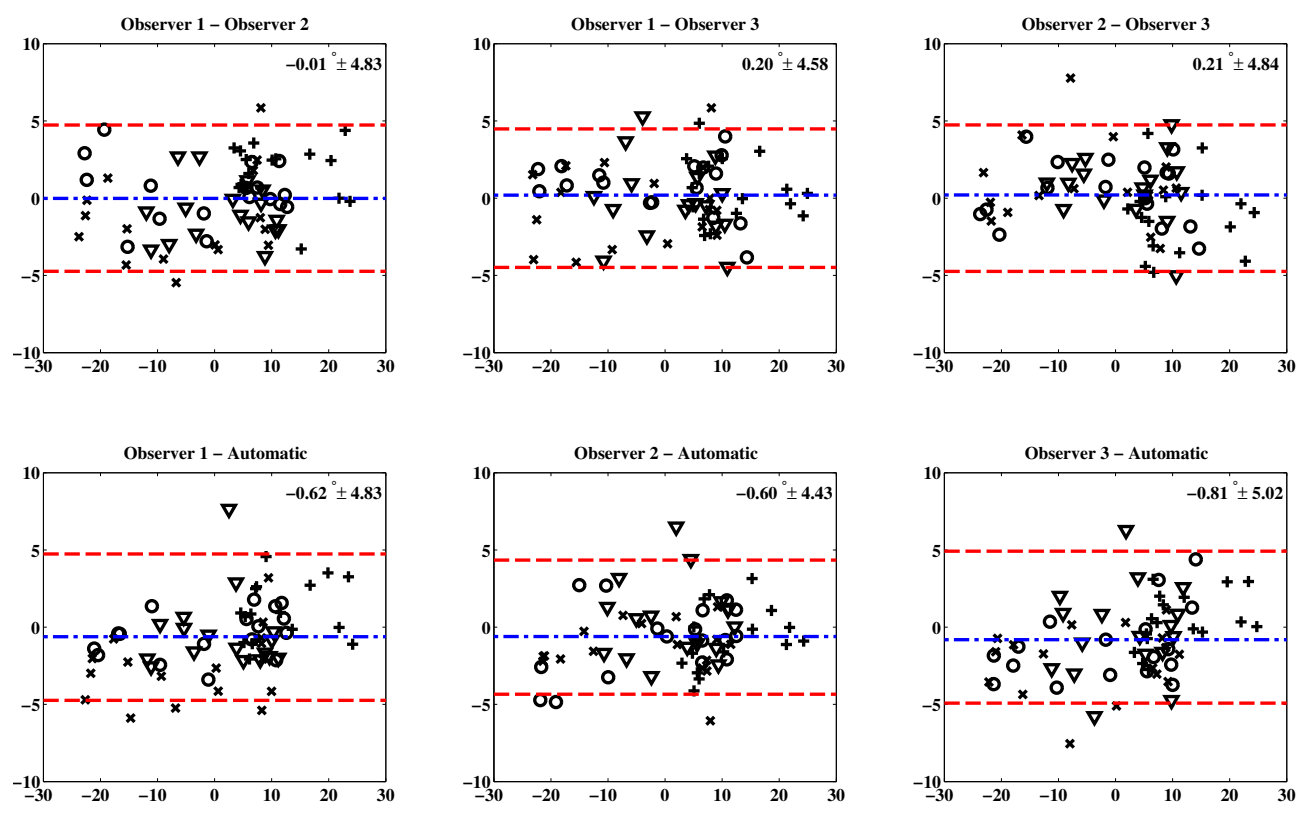

+ Patient 10 Patient $2 \times$ Patient $3 \nabla$ Patient 4

Figure 7. Bland-Altman plots (i.e. the difference between two measurements plotted against their mean) for measurements from observer 1, 2, 3 and automatic measurements. Additional horizontal lines correspond to the mean difference of the measurements (dotted) and to the lines of agreement (dashed-dotted), i.e. the $95 \%$ CI for the difference of the measurements. The plots reveal that no systematic difference is at hand when comparing the differences between manual and automatic measurements.

Table 1. Intraclass correlation coefficient using a two-way mixed model with absolute agreement, comparing the correlation in-between manual measurements and automatic measurements. The agreement in-between the different observers and the automatic method are all on a similar level, especially when considering the $95 \%$ CI.

\begin{tabular}{ccc}
\hline & ICC & $95 \%$ CI \\
\hline Automatic - Observer 1 & 0.971 & {$[0.953,0.982]$} \\
Automatic - Observer 2 & 0.979 & {$[0.966,0.987]$} \\
Automatic - Observer 3 & 0.970 & {$[0.951,0.981]$} \\
Observer 1 - Observer 2 & 0.980 & {$[0.967,0.987]$} \\
Observer 1 - Observer 3 & 0.983 & {$[0.972,0.989]$} \\
Observer 2 - Observer 3 & 0.980 & {$[0.967,0.987]$} \\
\hline
\end{tabular}

\section{Discussion}

In this paper, we have proposed a method for measurements of axial vertebral rotation, to be used for assessment of spinal deformity in idiopathic scoliosis. The presented method is the first computerized method that is fully automatic, which separates it from previously presented work on computerized methods. It also benefits from its computational speed, only requiring approximately ten to fifteen seconds when implemented in MATLAB, hence even further speed-ups are highly likely if 
Table 2. Average processing times for the different steps.

\begin{tabular}{lc}
\hline & Average processing time $[\mathrm{s}]$ \\
\hline Centreline extraction & $3.3 \pm 0.3$ \\
Disc detection & $0.1 \pm 0.0$ \\
Initial rotation estimation & $4.3 \pm 0.3$ \\
Centrepoint estimation & $0.6 \pm 0.1$ \\
Final rotation estimation & $4.3 \pm 0.3$ \\
\hline Total processing time & $12.6 \pm 0.7$ \\
\hline
\end{tabular}

implementing the same algorithm in $\mathrm{C} / \mathrm{C}++$. Table 2 provides a more detailed description of the processing times of the different steps of the proposed method.

The proposed automatic method was compared with the manual standard method of Aaro-Dahlborn for measuring AVR on 68 vertebrae. Both Bland-Altman plots and intraclass correlation coefficients show that the agreement between automatic and manual measurements are on the same level as the manual inter-observer agreement, thus, suggesting that the automatic method can confidently replace a manual method. A consistent, but minor decrease, in the ICC exists though, between comparing automatic measurements with manual and manual measurement with manual. However, these differences are likely to be due occasional inaccuracies in estimating the centrepoints of the vertebral bodies, hence causing a decrease in the accuracy of the estimated AVR.

Adam and Askin (2006) report overall mean differences and standard deviations of $-0.5^{\circ} \pm 3.3$ and $0.25^{\circ} \pm 3.8$ for 19 axial CT images and 204 reformatted axial $\mathrm{CT}$ images respectively, when comparing their proposed method with the method of Aaro-Dahlborn. Note that the reported standard deviations correspond to $95 \%$ CIs of $\pm 6.5^{\circ}$ and $\pm 7.4^{\circ}$ respectively. Vrtovec et al. (2010) report mean differences and $95 \%$ CIs of $-0.2^{\circ} \pm 2.6$ and $-0.3^{\circ} \pm 2.5$ for a $2 \mathrm{D}$ respectively a $3 \mathrm{D}$ implementation of their proposed method. These results where also obtained when comparing with the method of Aaro-Dahlborn, although on MRI data from two patients, one normal and one scoliotic. Thus, in comparison, our method provides a slightly larger mean difference than both Adam and Askin and Vrtovec et al., but with a smaller 95\% CI than Adam and Askin. Any more elaborate comparisons of the reported results are refrained from, since the different data sets and employed settings makes it difficult to compare. The results from Rogers et al. (2005) and Kouwenhoven et al. (2006) are not discussed here, since they do not compare their methods with any manual methods.

A limitation of the proposed method is its dependence on vertebral symmetry, both when finding the centrepoint of the vertebral bodies but more importantly when determining the rotation of the vertebral body. Asymmetry related to the vertebra itself, but also related to surrounding ribs, is believed to cause a decrease in the accuracy of the proposed method. However, at present there are no methods for measuring AVR that takes into account asymmetric vertebrae or how to measure the rotation of them. Thus, future research in this area is needed to develop methods, which do not rely on symmetrical vertebral bodies for defining how to measure AVR. Another limitation of the current method, is that the image data needs to have an initial resolution of $1 \times 1 \times 1 \mathrm{~mm}^{3}$ or less, the reason simply being that the method otherwise will fail when attempting to localize the discs between the vertebrae, which affects both the disc detection, the vertebra centrepoint estimation and the subsequent 
rotation estimation. Note that $\mathrm{CT}$ images are typically reconstructed to have a slice thickness of $3 \mathrm{~mm}$ or $5 \mathrm{~mm}$. This both limits the number of images to review for the radiologist and improves the SNR of the images. However, thinner slices, e.g. images with a slice thickness $<1 \mathrm{~mm}$, can easily be reconstructed from the same projection data as the thicker slices are reconstructed from. Because of this, there is no need for increased radiation dose in order to acquire thin slices.

A future expansion of the proposed method lies in its ability to estimate the pose (position and orientation) of each vertebra. In this work we have focused on the estimation of the AVR, but, as can be observed in figure 5 the method gives a position and orientation of each vertebra. This opens up for analysis schemes as described in for instance (Vrtovec et al. 2005, Illés et al. 2011) where not only the AVR of the end vertebrae and the apical vertebra are analysed, but the pose of each vertebra in the entire spine is considered.

\section{Acknowledgments}

This work was funded by the Swedish Research Council (grant 2007-4786) and the Swedish Foundation for Strategic Research (grant SM10-0022). Visualization and manual measurements were performed using MeVisLab (provided by Fraunhofer MEVIS, Bremen).

\section{References}

Aaro, S. and Dahlborn, M.: 1981, Estimation of vertebral rotation and the spinal and rib cage deformity in scoliosis by computer tomography, Spine 6(5), 460-467.

Adam, C. and Askin, G.: 2006, Automatic measurements of vertebral rotation in idiopathic scoliosis, Spine: an international journal for the study of the spine 31(3), E80-E83.

Cobb, R.: 1948, Outline for study of scoliosis, American Academy of Orthopaedic Surgeons, Instructional Course Lectures .

Glocker, B., Feulner, J., Criminisi, A., Haynor, D. R. and Konukoglu, E.: 2012, Automatic localization and identification of vertebrae in arbitrary field-of-view CT scans., in N. Ayache, H. Delingette, P. Golland and K. Mori (eds), Medical Image Computing and ComputerAssisted Intervention MICCAI 2012, Vol. 7512 of Lecture Notes in Computer Science, Springer, pp. 590-598.

Granlund, G. and Knutsson, H. (eds): 1995, Signal Processing for Computer Vision, Kluwer Academic Publishers, Dordrecht.

Hay, O., Hershkovitz, I. and Rivlin, E.: 2009, Spine curve modeling for quantitative analysis of spinal curvature, Engineering in Medicine and Biology Society, 2009. EMBC 2009. Annual International Conference of the IEEE, pp. 6356-6359.

Heidari, B., Fitzpatrick, D., McCormack, D. and Synnott, K.: 2006, Correlation of an induced rotation model with the clinical categorization of scoliotic deformity - a possible platform for prediction of scoliosis progression, Stud Health Technol Inform 123, 169-175.

Illés, T., Tunyogi-Csapó, M. and Somoskeöy, S.: 2011, Breakthrough in three-dimensional scoliosis diagnosis: significance of horizontal plane view and vertebra vectors, European Spine Journal 20, 135-143.

Kalra, M., Quick, P., Singh, S., Sandborg, M. and Persson, A.: 2012, Whole spine ct for evaluation of scoliosis in children: feasibility of sub-millisievert scanning protocol., Acta radiologica (Stockholm, Sweden: 1987).

Kim, Y. and Kim, D.: 2009, A fully automatic vertebra segmentation method using 3D deformable fences., Comp. Med. Imag. and Graph. 33(5), 343-352.

Klinder, T., Ostermann, J., Ehm, M., Franz, A., Kneser, R. and Lorenz, C.: 2009, Automated modelbased vertebra detection, identification, and segmentation in CT images, Medical Image Analysis 13(3), 471-482.

Kouwenhoven, J., Vincken, K., Bartels, L. and Castelein, R.: 2006, Analysis of pre-existent vertebral rotation in the normal spine, Spine 31(13), 1467-1472. 
Kuklo, T., Potter, B. and Lawrence, L.: 2005, Vertebral rotation and thoracic torsion in adolescent idiopathic scoliosis: What is the best radiographic correlate?, Journal of Spinal Disorders and Techniques 18(2), 139-147.

Lam, G., Hill, D., Le, L., Raso, J. and Lou, E.: 2008, Vertebral rotation measurement: a summary and comparison of common radiographic and CT methods, Scoliosis 3(1), 16.

Rogers, B., Haughton, V., Arfanakis, K. and Meyerand, E.: 2002, Application of image registration to measurement of intervertebral rotation in the lumbar spine, Magnetic Resonance in Medicine 48(6), 1072-1075.

Rogers, B., Wiese, S., Blankenbaker, D., Meyerand, E. and Haughton, V.: 2005, Accuracy of an automated method to measure rotations of vertebrae from computerized tomography data, Spine (Phila Pa 1976) 30(6), 694-696.

Skalli, W., Lavaste, F. and Descrimes, J.: 1995, Quantification of three-dimensional vertebral rotations in scoliosis: what are the true values?, Spine 20(5), 546-553.

Templin, C. R. and Sarwark, J. F.: 2008, Infantile and juvenile scoliosis, Thieme Medical Publishers Inc., pp. 569-579.

Vrtovec, T.: 2008, Modality-independent determination of vertebral position and rotation in 3D, Medical Imaging and Augmented Reality, Vol. 5128 of Lecture Notes in Computer Science, Springer Berlin Heidelberg, pp. 89-97.

Vrtovec, T., Likar, B. and Pernuš, F.: 2005, Automated curved planar reformation of 3D spine images, Physics in Medicine and Biology 50(19), 4527.

Vrtovec, T., Likar, B. and Pernuš, F.: 2007, Determination of 3D location and rotation of lumbar vertebrae in CT images by symmetry-based auto-registration, SPIE Medical Imaging 2007, Image Processing, 17-22 Feb, San Diego CA, USA, J.P.W. Pluim, J.M. Reinhardt (Eds.), Vol. 6512, p. 65121Q.

Vrtovec, T., Pernu, F. and Likar, B.: 2009, A review of methods for quantitative evaluation of axial vertebral rotation, European Spine Journal 18, 1079-1090.

Vrtovec, T., Pernuš, F. and Likar, B.: 2009, A review of methods for quantitative evaluation of spinal curvature, European Spine Journal 18(5), 593-607.

Vrtovec, T., Pernuš, F. and Likar, B.: 2010, Determination of axial vertebral rotation in MR images: comparison of four manual and a computerized method, European Spine Journal 19, 774-781.

Štern, D., Likar, B., Pernuš, F. and Vrtovec, T.: 2010, Automated detection of spinal centrelines, vertebral bodies and intervertebral discs in $\mathrm{CT}$ and MR images of lumbar spine, Physics in Medicine and Biology 55(1), 247.

Yao, J., O'Connor, S. and Summers, R.: 2006, Automated spinal column extraction and partitioning, Biomedical Imaging: Nano to Macro, 2006. 3rd IEEE International Symposium on, pp. 390393. 